\title{
FEM Simulation of Axisymmetric Pellet Injection System Using HTS Linear Acceleration*)
}

\author{
Teruou TAKAYAMA, Takazumi YAMAGUCHI, Ayumu SAITOH, Atsushi KAMITANI \\ and Hiroaki NAKAMURA ${ }^{1)}$ \\ Graduate School of Science and Engineering, Yamagata University, Yonezawa 992-8510, Japan \\ ${ }^{1)}$ National Institute for Fusion Science, Toki 509-5292, Japan
}

(Received 24 December 2018 / Accepted 13 March 2019)

\begin{abstract}
The acceleration performance of the pellet injection system used in a superconducting linear acceleration to fuel the nuclear fusion reactor has been investigated numerically. To this end, the numerical model has been developed for analyzing the shielding current density in a high-temperature superconducting (HTS) film by using the finite element method. By using the code, the pellet injection system has been simulated. The results of the computations show that, for the single acceleration coil, the final velocity increases almost in proportion to the height of the coil. For the multiple coils, the pellet container can be accelerated to $5 \mathrm{~km} / \mathrm{s}$ or more by about 6.87 seconds. Consequently, the length of the electromagnetic rails for a single coil becomes shorter than that for the multiple coils.
\end{abstract}

(C) 2019 The Japan Society of Plasma Science and Nuclear Fusion Research

Keywords: accelerator magnet, finite element analysis, linear accelerator, nuclear fuel, thin film

DOI: $10.1585 /$ pfr. 14.3401077

\section{Introduction}

As the fuel supply to the nuclear fusion reactor, a pellet injection system by using solid hydrogen pellets made of the frozen hydrogen/deuterium has been developed [1,2]. The solid hydrogen pellets are accelerated such as the air gun using the helium gas and driven into a hightemperature plasma. As a result, the melted pellets become high-density plasma clusters called plasmoid. In the case of the tokamak-type fusion reactor, the plasmoid reaches the plasma core by injecting the pellet from the inner side of the donut-shaped plasma. On the other hand, although the plasmoid phenomenon occurs in the helical-type fusion reactor, the plasmoid cannot reach the plasma core. Therefore, the fuel supply efficiency becomes low, and it is difficult to control the fusion combustion. For solving this problem, some papers have discussed the various possible solutions in the helical-type fusion reactor $[3,4]$.

In order to inject the ice pellets into the plasma core, a novel pellet injection system by using a Superconducting Linear Acceleration (SLA) is proposed recently. In this system, a pellet container attaching the HTS films for acceleration and levitation is accelerated like the magnetic levitation train electromagnetically. As a result, the estimation speed of the pellet injection system by the SLA becomes $5-10 \mathrm{~km} / \mathrm{s}$ or more. However, it is not clear how much pellet speed can be obtained because the SLA has not yet applied to the pellet injection system.

author'se-mail: takayama@yz.yamagata-u.ac.jp

*) This article is based on the presentation at the 27th International Toki Conference (ITC27) \& the 13th Asia Pacific Plasma Theory Conference (APPTC2018)

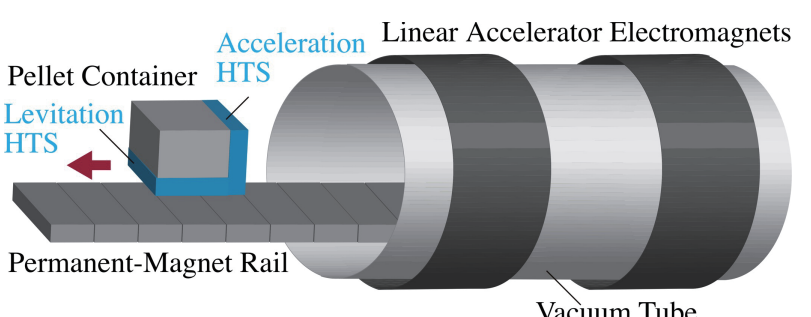

(a)

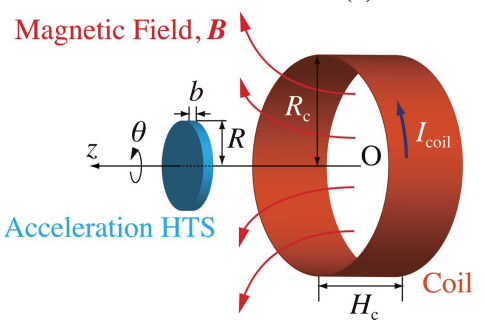

(b)

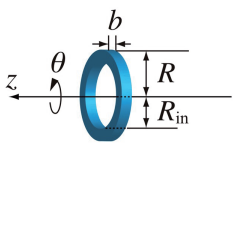

(c)
Fig. 1 A schematic views of (a) a pellet injection system used in the superconducting linear acceleration (SLA), (b) a pellet injection system used in the SLA for an axisymmetric model, and (c) a ring-shaped film.

The purpose of the present study is to develop the numerical code for analyzing the time evolution of the shielding current density by means of the finite element method (FEM). By using the code, we investigate the acceleration performance of the pellet injection system by the SLA.

\section{Governing Equation and Equation of Motion}

In a pellet injection system used in the superconduct- 
ing linear acceleration (SLA), an ice pellet with two types of an attached HTS film is accelerated on the electromagnetic rails and the linear accelerator electromagnets in the vacuum tube (see Fig. 1 (a)). After the pellet levitates by an electromagnetic force generated from the electromagnetic rails, it can be moved by the repulsive force acting between the electromagnets and the acceleration film. In the present study, we simulate the SLA pellet injection system by using the FEM. Note that we only use the acceleration HTS film in order to investigate the acceleration performance.

In Fig. 1 (b), a schematic view of a pellet injection system used in the SLA for an axisymmetric model. Throughout the present study, we adopt an acceleration coil of radius $R_{\mathrm{c}}$ and height $H_{\mathrm{c}}$. In addition, although we use a diskshaped HTS film of radius $R$ and thickness $b$, a ring-shaped film of an inner radius $R_{\text {in }}$ is also used in subsection 3.1. In order to evaluate a magnetic flux density $\boldsymbol{B}$ generated by the coil, a coil current $I_{\text {coil }}$ flows in it. By taking the symmetry axis as $z$-axis and choosing the centroid of the coil as the origin $\mathrm{O}$, we use the cylindrical coordinate $(r, \theta, z)$. In the following, the unit vectors along the $r-, \theta-$, and $z_{-}^{-}$ directions are $\boldsymbol{e}_{r}, \boldsymbol{e}_{\theta}$, and $\boldsymbol{e}_{z}$, respectively.

In the present study, we suppose the thin-layer approximation [5]: the shielding current density $\boldsymbol{j}$ can hardly flow in the thickness direction because the thickness of the HTS film is sufficiently thin. By using the above assumption, we can write the shielding current density $\boldsymbol{j}$ as follows: $\boldsymbol{j}=(2 / b) \nabla S \times \boldsymbol{e}_{z}$. In addition, the time evolution of a scalar function $S(r, t)$ is governed by the integrodifferential equations [5]:

$$
\begin{gathered}
\mu_{0} \frac{\partial}{\partial t} \int_{0}^{R} Q\left(r, r^{\prime}\right) S\left(r^{\prime}, t\right) r^{\prime} d r^{\prime}+\frac{2}{b} S \\
+\frac{\partial}{\partial t}\left\langle\boldsymbol{B} \cdot \boldsymbol{e}_{z}\right\rangle+\frac{1}{r} \frac{\partial}{\partial r} r\left(\boldsymbol{E} \cdot \boldsymbol{e}_{\theta}\right)=0,
\end{gathered}
$$

where $\boldsymbol{B}$ is an applied magnetic field generated by the acceleration coil, and a bracket \langle\rangle is an average operator over the thickness. Incidentally, the explicit form of the function $Q\left(r, r^{\prime}\right)$ is given in [5].

The shielding current density $\boldsymbol{j}$ and an electric field $\boldsymbol{E}$ are closely related through the $J-E$ constitutive equation $\boldsymbol{E}=E(|\boldsymbol{j}|)[\boldsymbol{j} /|\boldsymbol{j}|]$. As the function $E(j)$ characterizing the superconducting properties, we adopt the power law: $E(j)=E_{\mathrm{C}}\left[j / j_{\mathrm{C}}\right]^{N}$ Here, $j_{\mathrm{C}}$ and $E_{\mathrm{C}}$ are the critical current density and the critical electric field, whereas $N$ is an nonnegative integer.

In order to determine the dynamic motion of the acceleration HTS, the Newton's law of motion is given as follows:

$$
\frac{d^{2} z}{d t^{2}}=\frac{4 \pi}{m} \int_{0}^{R} \frac{\partial S}{\partial r}\left\langle B_{r}\right\rangle r d r,
$$

where $B_{r}(z, r)$ is $r$-component of an applied magnetic field $\boldsymbol{B}$ and $m$ is the total mass of the pellet container and the HTS film.

We apply the initial condition to (1) and (2) as follows: $S(r, 0)=v=0$ at $t=0$ and $z=z_{0}$ at $t=0$, where $z_{0}$ is an initial position of the HTS and $v(=d z / d t)$ is velocity of the acceleration film. In contrast, we use the boundary condition $S(R, t)=0$. When the initial-boundary-value problem of (1) and (2) is solved, the time evolution of the shielding current density and the dynamic motion of the HTS can be determined.

The initial-boundary-value problem of (1) and (2) is discretized with respect to space by using the FEM. The HTS region $\left[R_{\text {in }}, R\right]$ is equally divided so as to be the element size: $I_{\mathrm{e}}=R /(n-1)$, where $n$ denotes the number of the nodes in the FEM. As a result, the initial-boundary-value problem is reduced to the problem to solve the following simultaneous ordinary differential equations (ODEs):

$$
\frac{d s}{d t}=f(t, s)
$$

Here, $s$ and $f(t, s)$ are given by

$$
\boldsymbol{s}=\left[\begin{array}{c}
\boldsymbol{S} \\
v \\
z
\end{array}\right], \boldsymbol{f}(t, \boldsymbol{s})=\left[\begin{array}{c}
-W^{-1} U[\boldsymbol{e}(\boldsymbol{S})+v \boldsymbol{c}(z)+\boldsymbol{h}(z)] \\
\frac{4 \pi}{m} \boldsymbol{a}^{T}(z) \boldsymbol{S} \\
v
\end{array}\right],
$$

where symmetric matrix $W$ is defined by $W \equiv U W^{*} U+F$. The $n$-by- $n$ matrix $W^{*}$ is obtained from the function $Q\left(r, r^{\prime}\right)$ and FEM's shape functions [5]. Matrices $U$ and $F$ are determined from the boundary conditions. In addition, $S$ and $\boldsymbol{e}(\boldsymbol{S})$ are the nodal vectors corresponding to $S(r, t)$ and $\boldsymbol{E}$. The vectors $\boldsymbol{a}(z), \boldsymbol{c}(z)$ and $\boldsymbol{h}(z)$ are the nodal vectors corresponding to the applied magnetic field $\boldsymbol{B}$. In the present study, the ODEs (3) are solved by the Runge-Kutta method with the adaptive stepsize control.

\section{Simulation of Pellet Injection System by HTS}

On the basis of the method described in section 2, we developed the FEM code for analyzing both the time evolution of the shielding current density and the dynamic motion of the pellet container with an acceleration HTS film. By using the code, we investigate the acceleration performance of the pellet injection system by the SLA. Incidentally, the numerical computations are carried out on FUJITSU Supercomputer PRIMEHPC FX100 with double precision.

Throughout the present study, the geometrical and physical parameters are fixed as follows: $R_{\mathrm{c}}=5 \mathrm{~cm}, z_{0}=$ $1 \mathrm{~mm} . m=10 \mathrm{~g}, N=20, j_{\mathrm{C}}=1 \mathrm{MA} / \mathrm{cm}^{2}, E_{\mathrm{C}}=1 \mathrm{mV} / \mathrm{m}$, $R=4 \mathrm{~cm}, b=1 \mathrm{~mm}$, and $n=201$.

\subsection{Ring-shaped HTS}

In the previous study, although we have adopted the disk-shaped HTS (see Fig. 1 (b)), it is necessary to reduce the total mass of the pellet container to increase a pellet velocity. In the pellet injection system using the SLA, a shielding current density is known to localize near the film edge [6]. Therefore, we expect that the acceleration performance will be not degraded, even if there exists the hole 
centered on the origin in the HTS film, In this subsection from above background, let us investigate the acceleration performance by using not only a disk-shaped HTS but also a ring-shaped one (see Fig. 1 (c)). Especially, we examine the influence of the pellet velocity on the inner radius of the HTS. To this end, we use the single coil for accelerating the pellet, and the coil current $I_{\text {coil }}$ is given by $I_{\text {coil }}(t, z)=\alpha t(z \geq 0)$. Here, $\alpha$ is the increasing rate of the coil current and its value is fixed as $\alpha=20 \mathrm{kA} / \mathrm{ms}$.

Firstly, the typical cases of a shielding current density $j$ in a disk-shaped or a ring-shaped HTSs are shown as Fig. 2. We see from this figure that $j$-distributions for $R_{\text {in }}$ $=0 \mathrm{~m}$ and $R_{\text {in }}=2 \mathrm{~cm}$ are localized near the film edge of $r=R$. For $R_{\text {in }}=2 \mathrm{~cm}, j$ also flows near $r=R_{\text {in }}$ and its value tends to increase with the value of $R_{\text {in }}$.

In Fig. 3, we next show the time dependence of the pellet velocity $v$. We see from this figure that the velocity $v$ drastically increases for $5 \mathrm{~s} \lesssim t \lesssim 6 \mathrm{~s}$, whereas the value of $v$ becomes constant for $t \gtrsim 6 \mathrm{~s}$. The velocity $v$ for $R_{\text {in }}=$ $3.6 \mathrm{~cm}$ is slightly slower than that for $R_{\text {in }}=0 \mathrm{~m}$.

Finally, in order to investigate the acceleration performance quantitatively for the ring-shaped HTS, we use a final velocity $v_{\mathrm{f}}$. This value is determined from the $v-t$ curve where the velocity becomes constant. $v_{\mathrm{f}}$ is obtained as a function of the inner radius $R_{\text {in }}$ for the HTS and is depicted in Fig. 4. From this figure, the final velocity $v_{\mathrm{f}}$ becomes almost constant for $R_{\text {in }}<2 \mathrm{~cm}$. In other words, the hole for $0 \leq R_{\text {in }} \leq 2 \mathrm{~cm}$ hardly affects the acceleration performance

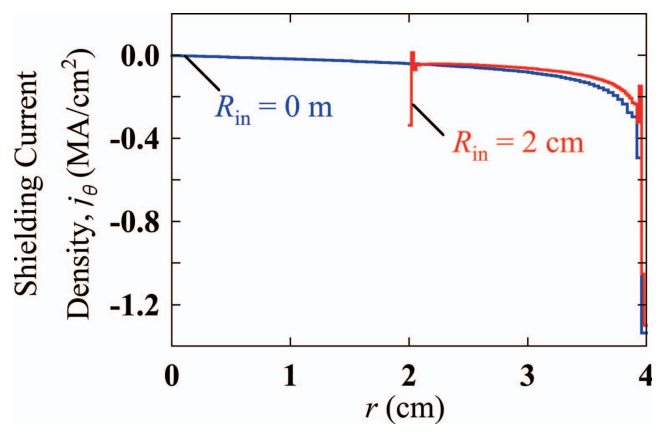

Fig. 2 The distributions of a shielding current density in the HTS film at time $t=5 \mathrm{~s}$. Here, $H_{\mathrm{c}}=10 \mathrm{~cm}$.

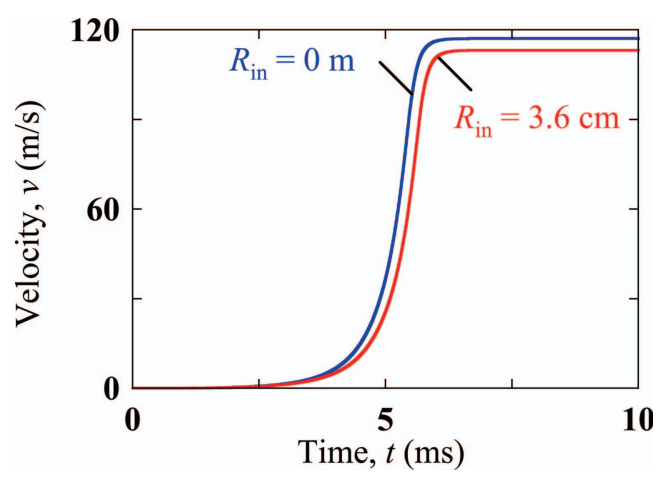

Fig. 3 Time dependence of the pellet velocity $v$ for the case with $H_{\mathrm{c}}=10 \mathrm{~cm}$. of the pellet injection system by the SLA. It is found that, for $2 \mathrm{~cm}<R_{\text {in }}$, the value of $v_{\mathrm{f}}$ monotonously decreases with increasing $R_{\text {in }}$. Therefore, even when there exists a hole in the acceleration HTS, the same speed as without a hole can be obtained. In this sense, this result implies that the mass of the pellet container can be reduced.

\subsection{Improvement of acceleration performance}

As shown in Fig. 4 , we obatin the final velocity $v_{\mathrm{f}}=$ $117 \mathrm{~m} / \mathrm{s}$ of the pellet injection system using the SLA. However, it is far from $v=5 \mathrm{~km} / \mathrm{s}$ which is the pellet velocity reaching the plasma core. In this subsection, let us discuss the improvement of the acceleration performance by the following two methods.

- increase the length of a single acceleration coil

- use the multiple coils

Moreover, we adopt the disk-shaped HTS (i.e. $R_{\text {in }}=0 \mathrm{~m}$ ) in this subsection.

In Fig. 5, we firstly show the dependence of the final velocity $v_{\mathrm{f}}$ of the height $H_{\mathrm{c}}$ of the coil. As a result, we found that the final velocity $v_{\mathrm{f}}$ increases almost in proportion to the height $H_{\mathrm{c}}$ of the coil. Moreover, a regression line can be obtained from the relationship between $v_{\mathrm{f}}$ and $H_{\mathrm{c}}$ as follows: $v_{\mathrm{f}}=39.5 H_{\mathrm{c}}^{0.45}$. Hence, it is possible to estimate the velocity $v$ of $H_{\mathrm{c}}=1 \mathrm{~m}$ or more. Conse-

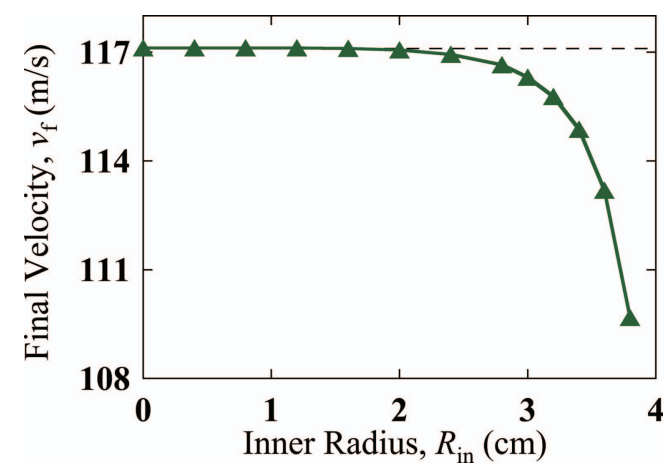

Fig. 4 Dependence of the final velocity $v_{\mathrm{f}}$ on the inner radius $R_{\text {in }}$ for the case with $H_{\mathrm{c}}=10 \mathrm{~cm}$.

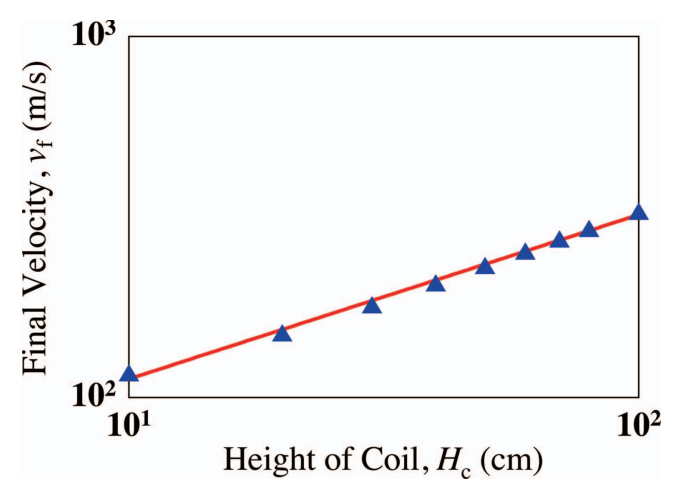

Fig. 5 Dependence of the final velocity $v_{\mathrm{f}}$ on the height $H_{\mathrm{c}}$ of the coil. 


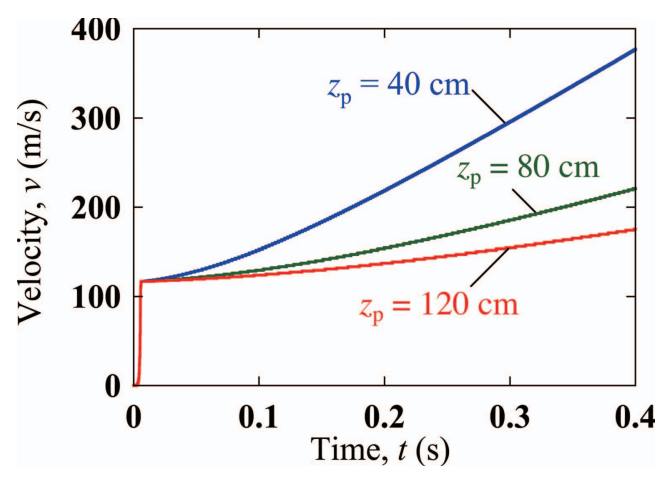

Fig. 6 Time dependence of the pellet velocity $v$ for the case with $H_{\mathrm{c}}=10 \mathrm{~cm}$.

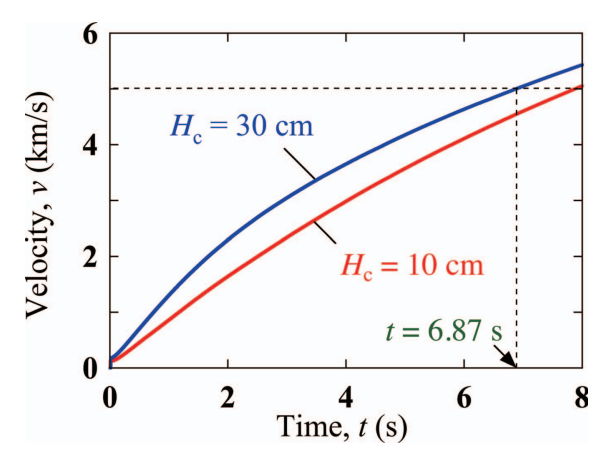

Fig. 7 Time dependence of the velocity $v$ for $H_{\mathrm{c}}=10 \mathrm{~cm}$ and $H_{\mathrm{c}}$ $=30 \mathrm{~cm}$.

quently, substituting the final velocity $v_{\mathrm{f}}=5 \mathrm{~km} / \mathrm{s}$ in the above equation, we can obtain $H_{\mathrm{c}}=470 \mathrm{~m}$.

In the following, we use the multiple coils to accelerate the pellet container with the HTS film. Moreover, we can determine the $z$-coordinate as the position of the acceleration HTS for the multiple coils by using the following modulo operator: $z_{\bmod }=\bmod \left(z+z_{\mathrm{p}} / 2, z_{\mathrm{p}}\right)-z_{\mathrm{p}} / 2$. As a result, the dynamic motion of the HTS film can be looped to produce a continuous in the range $-z_{\mathrm{p}} / 2 \leq z_{\bmod } \leq z_{\mathrm{p}} / 2$. Also, the coil current is written as follows:

$$
I_{\text {coil }}\left(t, z_{\text {mod }}\right)=\left\{\begin{array}{ll}
\alpha\left(t-t_{\text {min }}\right) & \left(0 \leq z_{\text {mod }} \leq z_{\text {limit }}\right) \\
0 & (\text { otherwise })
\end{array} .\right.
$$

Here, $t_{\min }$ denotes the time at $z_{\bmod }=0 \mathrm{~m}$. Also, a range $0 \leq$ $z_{\text {mod }} \leq z_{\text {limit }}$ in (4) denotes an acceleration range, where $z_{\text {limit }}$ is the limit of the range.

Figure 6 shows the velocity-time graph of the pellet injection system using the SLA for various cases of the coil interval $z_{\mathrm{p}}$. From this figure, the velocity $v$ drastically increases by the 1st coil, and subsequently, the pellet is gradually accelerated after the 2 nd coil. We obtain the values of $v$ at time $t=0.4 \mathrm{~s}$ for $z_{\mathrm{p}}=40 \mathrm{~cm}, z_{\mathrm{p}}=80 \mathrm{~cm}$, and $z_{\mathrm{p}}=120 \mathrm{~cm}$ are $v=377 \mathrm{~m} / \mathrm{s}, v=221 \mathrm{~m} / \mathrm{s}$, and $v=176 \mathrm{~m} / \mathrm{s}$, respectively. Consequently, as the coil interval decreases, the pellet speed increases dramatically. In the following, the coil interval $z_{\mathrm{p}}$ is fixed as $z_{\mathrm{p}}=40 \mathrm{~cm}$.

Let us finally investigate the acceleration time required for the velocity $v=5 \mathrm{~km} / \mathrm{s}$ or more for reaching the plasma core. In Fig. 7, we show the time dependence of the pellet velocity $v$ from $0 \mathrm{~s} \leq t \leq 8 \mathrm{~s}$. From this figure, we found that, for $H_{\mathrm{c}}=10 \mathrm{~cm}$ and $H_{\mathrm{c}}=30 \mathrm{~cm}$, the velocity $v=5 \mathrm{~km} / \mathrm{s}$ or more requires time $t \gtrsim 7.87 \mathrm{~s}$ and $t \gtrsim 6.87 \mathrm{~s}$. In addition, the required distance of the electromagnetic rails becomes about $21 \mathrm{~km}$ for $H_{\mathrm{c}}=30 \mathrm{~cm}$.

\section{Conclusions}

Conclusions obtained in the present study are summarized as follows:

1. If the inner radius is half of the outer radius for the ring-shaped acceleration HTS, the pellet velocity is hardly degraded. In this sense, this result implies that the mass of the pellet container can be reduced.

2. For the single acceleration coil, the final velocity increases almost in proportion to the height of the coil. From the relationship between the final velocity and the height of the coil, we obtain the height $H_{\mathrm{c}}=470 \mathrm{~m}$ of the coil to reach the velocity $v=5 \mathrm{~km} / \mathrm{s}$.

3 . For the multiple coil, the pellet container can be accelerated to $5 \mathrm{~km} / \mathrm{s}$ or more for about 6.87 seconds. As a result, the required distance of the electromagnetic rails becomes about $21 \mathrm{~km}$.

Consequently, although it is necessary to make the shape of the rail not straight but circular, the length of the electromagnetic rails for a single coil becomes shorter than that for the multiple coils.

\section{Acknowledgment}

A part of this work was also carried out with the support and under the auspices of the NIFS Collaboration Research program (NIFS18KECA057 and NIFS18KNTS056). Moreover, the numerical computations were carried out on Fujitsu PRIMEHPC FX100 of the LHD Numerical Analysis Server in NIFS.

[1] R. Sakamoto, H. Yamada and LHD experimental group, Plasma Fusion Res. 4, 002 (2009).

[2] R. Sakamoto, G. Motojima, H. Hayashi, T. Inoue, Y. Ito, H. Ogawa, S. Takami, M. Yokota and H. Yamada, Rev. Sci. Instrum. 84, 083504 (2013).

[3] R. Sakamoto, J. Miyazawa, H. Yamada, S. Masuzaki, A. Sagara and the FFHR Design Group, Nucl. Fusion 52, 083006 (2012).

[4] R. Sakamoto and H. Yamada, IEEE Trans. Plasma Sci. 44, 2915 (2016).

[5] A. Kamitani and S. Ohshima, IEICE Trans. Electron. E82-C, 766 (1999).

[6] T. Takayama, A. Saitoh and A. Kamitani, IEEE Trans. Appl. Supercond. 28, 3700205 (2018). 